Int. J. Odontostomat., 10(1):75-84, 2016.

\title{
Expresión Génica del Factor de Crecimiento Transformante Beta en Niños con Fisura Labio Palatina no Sindrómica
}

\author{
Gene Expression of Transforming Growth Factor Bet \\ in Children with Non-Syndromic Cleft Lip and Palate
}

\author{
Meisser Vidal Madera Anaya*; Farith González Martinez*; \\ Daniel Esteban Romero Suarez ${ }^{* * *} \&$ Amileth Suárez Causado ${ }^{* \star * *}$
}

MADERA, A. M. V.; GONZÁLEZ, M. F.; ROMERO, S. D. E. \& SUÁREZ, C. A. Expresión génica del factor de crecimiento Transformante Beta en niños con fisura labio palatina no sindrómica. Int. J. Odontostomat., 10(1):75-84, 2016.

RESUMEN: El objetivo fue determinar la expresión génica del factor de crecimiento transformante beta (TGF-ß) y posibles factores de riesgo en niños con y sin fisuras labio palatina no sindrómicas (FLPNS). Diseño de casos (Niños con fisuras orales; $n=20$ ) y controles (niños no afectados; $n=40$ ). A partir de muestras de saliva se realizó la extracción de mRNA utilizando el RNeasy ${ }^{\circledR}$ Protect Saliva Mini Kit-QIAgen y la expresión génica del TGF-ß mediante la reacción en cadena de la polimerasa en transcriptasa reversa, además se aplicó un cuestionario para registrar características sociodemográficas y posibles factores de riesgo durante el periodo de concepción (medicamentos, enfermedades, alcohol, cigarrillo y edad de los padres). Los datos fueron analizados mediante medidas de tendencia central y dispersión, frecuencias y porcentajes, para establecer asociaciones se utilizaron las prueba T-Student, Chi-cuadrado y Odds ratio, asumiendo un límite de confianza de 0,05. El promedio de edad de los participantes fue de 6,8 (DE= 4,6) años y el 63,3 \% eran de sexo masculino. Al evaluar los posibles factores asociados con el desarrollo de FLPNS no se encontraron diferencias significativas, sin embargo el $11,7 \%$ de las madres habían ingerido algún tipo de medicamentos durante el embarazo, el 1,7 \% habían fumado algún cigarrillo y el 13,3 \% ingerido alcohol. Existieron diferencias significativas en la expresión génica del TGF- $ß$ entre los grupos $(p=0,0205)$, siendo menor en grupo de casos. Los factores de riesgo evaluados mostraron poca evidencia de asociación con la presencia de FLAPNS, los niños con esta alteración tienen menor expresión génica del TGF-ß, sugiriendo que alteraciones moleculares en la vía de señalización del TGF-ß posiblemente están involucradas en el desarrollo de las fisuras labio palatinas, ya que se pueden afectar procesos de diferenciación, crecimiento y proliferación celular, en donde participan varios genes incluyendo el TGF-ß.

PALABRAS CLAVE: fisura labio palatina, factor de crecimiento transformante beta, señalización celular.

\section{INTRODUCCIÓN}

Las fisuras labio palatinas (FLP) se generan por la falta de fusión de los tejidos del labio o del paladar durante las primeras etapas del desarrollo fetal, se encuentran entre las deformidades cráneo facial más frecuente y hacen parte de los defectos congénitos más comunes causados por el desarrollo facial anormal durante la gestación (Berbert-Campos et al., 2007; Cooper-Brown et al., 2008; Chen et al., 2008). La incidencia mundial de FLP es aproximadamente 1 a 2 por 1000 nacidos vivos, esta frecuencia varía en relación

Odontólogo, Magíster en Bioquímica, Universidad de Cartagena. Magíster en Epidemiología Clínica, Universidad de la Frontera, Temuco, Chile.

* Odontólogo, Especialista en Investigación Social, Magister en Salud Pública, Doctor en Toxicología Ambiental (c), Docente Facultad de Odontología, Universidad de Cartagena, Cartagena, Colombia.

*** Biólogo- Joven Investigador Prometeus\&Biomedicina aplicada a las ciencias clínicas. Facultad de Medicina, Universidad de Cartagena, Cartagena, Colombia.

**** Directora grupo Prometeus\&Biomedicina aplicada a las ciencias clínicas. Química Farmacéutica, Doctora en Bioquímica y Biología molecular, Docente Facultad de Medicina, Universidad de Cartagena, Cartagena, Colombia. 
al origen étnico, el área geográfica y a la naturaleza propia de la fisura (Prahl-Andersen \& Ju, 2006). En el contexto de las fisuras labiales extendidas a paladar, la incidencia es de aproximadamente 0,3 por cada 1000 en poblaciones afroamericanas, de 1,0 por cada 1000 en poblaciones caucásicas y de 2 a 3 por cada 1000 en poblaciones asiáticas; las fisuras unilaterales son nueve veces más comunes que las fisuras bilaterales y los hombres son en su mayoría más afectados por fisuras labiales en una relación de 2:1 respecto a las mujeres; mientras que estas son más afectadas por fisuras palatinas (Aminpour \& Tollefon, 2008; Costello \& Ruiz, 2009).

Las FLP constituyen uno de los principales problemas morfológicos, funcionales y estéticos; afectando la masticación, deglución, amamantamiento, digestión, habla, audición, la ventilación del oído medio y el desarrollo a nivel social (Chai \& Maxson, 2006; Ocak et al., 2013). Estos individuos requieren cuidados desde su nacimiento hasta la adultez de muchas disciplinas incluyendo enfermería, cirugía plástica, cirugía maxilofacial, otorrinolaringología, fonoaudiología, psicología, consejería genética y tratamiento odontológico (Wong \& Hagg, 2004; Jugessur et al., 2009; Mossey, et al., 2009; Ajike et al., 2013; Borno, et al., 2014)

La etiología de estas fisuras no se encuentra totalmente aclarada, sin embargo se intenta explicar por medio de un modelo de umbral multifactorial, en el cual se plantea que es producto de la interacción de factores endógenos y ambientales, entre los cuales están la etnia, variaciones geográficas, desordenes genéticos, aberraciones cromosómicas y exposición a teratógenos como el alcohol, tabaco, vitaminas, anticonvulsivantes y metales pesados; lo que sugiere que múltiples genes y factores ambientales esta involucrados en el desarrollo de las FLP (Prescott et al., 2001; Cobourne, 2004; Little et al., 2004; Lidral et al., 2008). Cerca del $70 \%$ de todas las fisuras orofaciales son no sindrómicas (fisuras labiopalatinas no sindrómicas -FLPNS-), mientras que aproximadamente el 30\% restante se presentan en compañía de síndromes como es el caso de los síndromes de Pai, Van der Woude, Marfán, Smith-Lemli-Opitz y LoeysDietz (Mazzieri et al., 2005; Scapoli et al., 2008; Meng et al., 2009).

Con relación a las FLPNS se ha reportado que algunos genes como el TGF- $\beta$, MSX1 (Msh homeobox 1), IRF6 (Interferon regulatorio del factor 6) y GABA (Ácido gamma-aminobutírico) muestran gran susceptibilidad para el desarrollo de estas fisuras (Massague
\& Wotton 2000; Chen et al., 2002; Sakaki-Yumoto et al., 2013). El TGF-ß es miembro de una superfamilia de factores polipeptídicos que estimulan varias redes de señalización involucradas en la determinación, crecimiento y diferenciación de un gran número de tipos celulares, particularmente células epiteliales (Shen, 2007; Kruithof-de Julio et al., 2011). Se han descrito diferentes clases de receptores de la familia del TGF$ß$, entre los que destacan el TßRI (Receptor beta tipo I) y el TßRII (Receptor beta tipo II), que tienen actividad serina-treonina proteína quinasa en su dominio citoplasmático y forman heterodímeros para ser activos (Andrieux et al., 2012; Tsai et al., 2013). El receptor TßRII tiene una actividad quinasa constitutiva, la unión del TGF- $ß$ al receptor TßRII es reconocida por el receptor TßRI, uniéndose a él para formar un complejo. A continuación, el receptor TßRI es fosforilado por el TßRII, lo que estimula su actividad serinatreonina quinasa, con la subsiguiente fosforilación de distintos miembros de la familia de proteínas Smad. Estas proteínas fosforiladas se unen a otro miembro de la familia, el Smad 4, como resultado se produce su translocación al núcleo, donde interacciona de forma célula específica con otros factores de transcripción, para finalmente regular la expresión génica. Se ha demostrado que el TGF- $ß$ inhibe las actividades de los complejos ciclina D-Cdk4/6 y ciclina E/Cdk2, lo que conduce a la hipofosforilación de $p$-Retinoblastoma y a una disminución de la actividad transcripcional de los efectores de factores de transcripción (Qian et al., 1997; Kang et al., 2009).

El objetivo de este estudio fue determinar la expresión génica del TGF-ß y posibles factores de riesgo en niños con y sin FLPNS.

\section{MATERIAL Y MÉTODO}

Tipo de estudio. Analítico de casos y controles, en donde los participantes fueron convocados de las clínicas odontológicas de la Universidad de Cartagena. Este estudio fue aprobado por el comité de ética de la Universidad de Cartagena.

Selección de muestra. El tamaño de la muestra se calculó en el programa Epi-info a partir de un error tipo I del $5 \%$, poder del $80 \%$ y frecuencia esperada de exposición en el grupo control del $26,3 \%$, para una relación de 1:2 el resultado mínimo requerido fue de 60 niños, 20 casos y 40 controles. Se denominaron casos a los niños con FLPNS, evaluándo- 
los a través de las características clínicas de esta alteración y mediante un cuestionario y examen físico que contemplaba excluir 100 características compatibles a síndromes; asimismo se les solicitó a los padres información personal que corroborara la presencia o no de algún síndrome. Estos fueron seleccionados a partir de los siguientes criterios: familias con historia residencial en el departamento de Bolívar durante el tiempo de la concepción del niño y que aceptaran participar autorizando la donación de muestras de saliva. Se denominaron como controles a los niños clínicamente saludables que no presentaban FLPNS, estos fueron seleccionados y emparejadosteniendo en cuenta la edad, sexo y municipio de residencia durante el tiempo de la concepción del niño. Para ambos grupos se excluyeron niños con antecedentes personales de enfermedades neoplásicas, inmuno-deficiencias, desordenes autoinmunes y hepatitis. Para que los niños pudieran participar era necesario que alguno de sus padres aceptara a partir de la firma del consentimiento informado por escrito.

Procedimientos. Se diseñó un cuestionario para obtener información relacionada con las características sociodemográficas y algunos posibles factores asociados (1. Edad, 2. Sexo, 3. Estrato socioeconómico, 3. Procedencia, 4. Consumo de alcohol durante el embarazo, 5. Ingesta de medicamentos durante el embarazo, 6. Consumo de cigarrillo durante el embarazo, 7. Enfermedades graves durante el primer trimestre de embarazo, 8. Edad de concepción de la madre, 9. Edad de concepción del padre). El profesional encargado de la recolección de esta información fue diferente al que realizó el examen clínico y pruebas moleculares.

Examen clínico odontológico. Se realizó por un odontopediatra calibrado en la identificación y clasificación de FLP. Para éste se utilizó luz artificial y espejo No. 5 , además era obligatoria la palpación del paladar y labio para los dos grupos, con el fin de evitar sesgos de selección. El proceso se inició observando el labio, detallando mucosa y detectando visualmente afecciones; después se procedió a palpar e inspeccionar el paladar desde la zona posterior al tercio anterior y de derecha a izquierda. Con este se evaluó la presencia y el tipo de fisura teniendo en cuenta la clasificación de Kernahan \& Stark (1958).

Obtención de las muestras de saliva. Las muestras de saliva humana no estimulada se colectaron en tubos de polipropileno en hielo, en un cantidad de $3 \mathrm{~mL}$. A todos los niños se les indicó el lavado de los dientes y la no ingesta de bebidas ni comida una hora antes de la colección salival. Todas las muestras se obtuvieron siguiendo el protocolo de salivación autónoma dentro del laboratorio de Bioquímica de la Universidad de Cartagena, donde fueron almacenadas a - 80 ${ }^{\circ} \mathrm{C}$ hasta su posterior análisis.

Extracción de ARN en saliva. En este se empleó el RNeasy ${ }^{\circledR}$ Protect Saliva Mini Kit de la marca Qiagen, siguiendo las recomendaciones del fabricante. La valoración de la concentración y el grado de pureza del ARN fue realizada por espectrofotometría a 230, 260 y $280 \mathrm{~nm}$ en el equipo NanoDrop 2000 UV-Vis Spectrophotometer (Thermo Scientific).

\section{Análisis de la expresión génica mediante RT-PCR. El ARN total se empleó para obtener el cDNA, el cual se utilizó para la amplificación por PCR convencional utilizando los "primers" correspondientes al GUSB y TGFß (Tabla I), dNTPs (10 mM), MgCl2 (25 mM) y 1 unidad de la polimerasa Ecotaq. La reacción de PCR se realizó en un termociclador (BIO-RAD T100TM) bajo las siguientes condiciones: $94^{\circ} \mathrm{C}$ durante 5 min, 40 ciclos a: $94{ }^{\circ} \mathrm{C}$ durante $30 \mathrm{~s}, 62,5^{\circ} \mathrm{C}$ durante $30 \mathrm{~s}, 72$ ${ }^{\circ} \mathrm{C}$ durante 1 min y un tiempo de elongación final a 72 ${ }^{\circ} \mathrm{C}$ durante $10 \mathrm{~min}$. Los productos de PCR se visualizaron mediante electroforesis en geles de agarosa al 1,0 \% en tampón TAE 1X (Tris 40 mM; 0,1 $\%$ Ácido acético glacial; EDTA $1 \mathrm{mM})$ conteniendo EZ- Vision $^{\circledR}\left(\right.$ Amresco ${ }^{\circledR}$ ) y exposición a la luz UV en el equi- po ChemiDoc ${ }^{\text {Th }} \mathrm{XRS}+$ System. Todas las muestras fue- ron analizadas por triplicado.}

Tabla I. Características de los Primers.

\begin{tabular}{|c|c|c|c|c|c|}
\hline Gen & Secuencia 5' 3' (Forward//Reverse) & Descripción & pb & $\operatorname{Tm}{ }^{\circ} \mathrm{C}$ & ID-Gen Bank \\
\hline GUSB & $\begin{array}{l}\text { ATCACCGTCACCACCAGCGT } \\
\text { GTCCCATTCGCCACGACTTTGT }\end{array}$ & $\begin{array}{l}\text { Glucoronidasa Beta, codifica una } \\
\text { hidrolasa que degrada } \\
\text { glicosaminoglicanos. Gen de } \\
\text { referencia. }\end{array}$ & 131 & 70,3 & M65002.1 \\
\hline TGFB & $\begin{array}{l}\text { GGCAGCTGTACATTGACTTCC } \\
\text { CCTTGCTGTACTGCGTGTCC }\end{array}$ & $\begin{array}{l}\text { Factor de crecimiento } \\
\text { transformante Beta. }\end{array}$ & 129 & 72,5 & M60316.1 \\
\hline
\end{tabular}

bp: pares de bases del tamaño del producto; Tm: temperatura de anillamiento 
Análisis estadístico. Se diseñó de una base de datos en Excel Microsoft ${ }^{\circledR}$ office 2010, luego esta fue transportada al programa STATA ${ }^{\circledR}$ (Stata Corp. LP, College Station, TX, USA). Inicialmente se evaluó el supuesto de normalidad de los datos a partir del test de Shapiro Wilk. Para el análisis descriptivo fueron usadas las medidas de tendencia central, dispersión y las proporciones. Para analizar la asociación entre las variables se utilizó la prueba T-Student para variables cuantitativas y la prueba Chi-cuadrado para las variables cualitativas; además se calculó la razón de oportunidades (Odds ratio). Para todas las pruebas se asumió una probabilidad límite de decisión de 0,05.

\section{RESULTADOS}

El promedio de edad de los participantes fue de 6,8 (DE= 4,6) años, en el cual la edad mínima fue de 3 meses y la máxima de 15 años. De manera general el $63,3 \%$ eran de sexo masculino y el $36,7 \%$ femeni- no, el $88,3 \%$ pertenecían al estrato 1 correspondiente a un nivel socioeconómico bajo, el $91,7 \%$ eran procedentes de la ciudad de Cartagena y el $8,3 \%$ restante provenían de otros municipios del departamento de Bolívar entres los que se encuentran San Jacinto, Cicuco, Santa Rosa, María La Baja y Santa Cruz de Mompox, al comparar las características sociodemográficas por los grupos no se encontraron diferencias significativas (Tabla II).

Con relación a los hallazgos identificados en el examen estomatológico se encontró que el tipo de fisura labio alvéolo palatina de mayor frecuencia fue la fisura bilateral completa con el $50 \%$, la cual se caracteriza por afectar los dos lados faciales comprometiendo tanto el paladar primario como el secundario, seguida de unilateral completa con el $35 \%$ y submucosa con el $15 \%$.

Al evaluar los posibles factores asociados con el desarrollo de FLPNS, se encontró que el $11,7 \%$ de las madres habían ingerido algún tipo de medicamentos

Tabla II. Distribución de las características sociodemográficas de los participantes.

\begin{tabular}{llcccc}
\hline & & Total participantes & Casos & Controles & \multirow{2}{*}{ Valor p } \\
\cline { 3 - 5 } & & $\mathbf{n}$ (columna \%) & $\mathbf{n}($ filas \%) & n (filas\%) & \\
\hline Total muestra & & $60(100)$ & $20(33,3)$ & $40(66,7)$ & \\
Sexo & Masculino & $38(63,3)$ & $14(36,8)$ & $24(63,2)$ & 0,449 \\
\multirow{2}{*}{ Estrato } & Femenino & $22(36,7)$ & $6(27,3)$ & $16(72,7)$ & \\
& 1 & $53(88,3)$ & $16(30,2)$ & $37(69,8)$ & \\
& 2 & $3(5,0)$ & $3(100,0)$ & $0(0,0)$ & 0,052 \\
\multirow{2}{*}{ Procedencia } & 3 & $4(6,7)$ & $1(25,0)$ & $3(75,0)$ & \\
& Cartagena & $55(91,7)$ & $17(30,9)$ & $38(69,1)$ & 0,186 \\
& Otro & $5(8,3)$ & $3(60,0)$ & $2(40,0)$ & \\
\hline
\end{tabular}

*Estadísticamente significativo, prueba Chi-cuadrado

Tabla III. Factores asociados con el desarrollo de fisuras labiopalatinas no sindrómicas (FLPNS).

\begin{tabular}{|c|c|c|c|c|c|c|}
\hline & & $\begin{array}{c}\text { Total participantes } \\
\text { n (columna \%) }\end{array}$ & $\begin{array}{c}\text { Casos } \\
\text { n (filas \%) }\end{array}$ & $\begin{array}{c}\text { Controles } \\
\text { n (filas } \%)\end{array}$ & OR & Valor $p$ \\
\hline Total muestra & & $60(100)$ & $20(33,3)$ & $40(66,7)$ & & \multirow{3}{*}{0,569} \\
\hline \multirow{2}{*}{ Ingesta de medicamentos } & Si & $7(11,7)$ & $3(42,9)$ & $4(57,1)$ & \multirow[b]{2}{*}{1,59} & \\
\hline & No & $53(88,3)$ & $17(32,1)$ & $36(67,9)$ & & \\
\hline \multirow[t]{2}{*}{ Consumo de cigarrillo } & $\mathrm{Si}$ & $\begin{array}{c}1(1,7) \\
59(983)\end{array}$ & $\begin{array}{l}1(100,0) \\
19(32\end{array}$ & $\begin{array}{c}0(0,0) \\
40(678)\end{array}$ & --- & 0,153 \\
\hline & $\mathrm{Si}$ & $8(13,3)$ & $1(12,5)$ & $7(87,5)$ & \multirow{2}{*}{0,25} & \multirow{2}{*}{0,179} \\
\hline Consumo de alcohol & No & $52(86,7)$ & $19(36,5)$ & $33(63,5)$ & & \\
\hline Enfermedad primer trimestre & $\begin{array}{l}\mathrm{Si} \\
\mathrm{No}\end{array}$ & $\begin{array}{c}1(1,7) \\
59(98,3)\end{array}$ & $\begin{array}{l}1(100,0) \\
19(32,2)\end{array}$ & $\begin{array}{c}0(0,0) \\
40(67,8)\end{array}$ & --- & 0,153 \\
\hline \multirow{2}{*}{ Edad de œncepción madre } & > 35 años & $8(13,3)$ & $1(12,5)$ & $7(87,5)$ & \multirow{2}{*}{0,25} & \multirow{2}{*}{0,179} \\
\hline & $\leq 35$ años & $52(86,7)$ & $19(36,5)$ & $33(63,5)$ & & \\
\hline Edad de concepción padre & $\begin{array}{l}>40 \text { años } \\
\leq 40 \text { años }\end{array}$ & $\begin{array}{c}5(8,3) \\
55(91,7)\end{array}$ & $\begin{array}{c}3(60,0) \\
17(30,9)\end{array}$ & $\begin{array}{c}2(40,0) \\
38(69,1)\end{array}$ & 3,35 & 0,186 \\
\hline
\end{tabular}

*Estadísticamente significativo, prueba Chi-cuadrado 
durante el embarazo, entre los que se reportaron están los antibióticos y antihipertensivos, el 1,7\% habían consumido algún cigarrillo, el 13,3\% habían consumido alcohol, el 98,3\% negaron cualquier tipo de enfermedad durante el primer trimestre de embarazo, el $86,7 \%$ de las madres eran menores de 35 años al momento de la concepción del niño y el $91,7 \%$ de los padres eran menores de 40 años al momento de la concepción del niño, al comparar los factores asociados por los grupos no se encontraron diferencias significativas (Tabla III).

Para la evaluación de la expresión génica se utilizó como gen normalizador el GUSB, el cual se expresó tanto en casos como en controles, al comparar la expresión y los niveles de expresión de este gen con cada uno de los grupos de estudio no se encontraron diferencias estadísticamente significativas $(p=0,1157)$. Para el gen TGF- $ß$ existieron diferencias significativas en la expresión génica entre los grupos $(p=0,0205)$, siendo mayor en grupo control, lo que sugiere que la disminución en la expresión génica del TGF-ß posiblemente disminuye la respuesta celular en la vía de señalización del TGF$ß$ lo que podría generar durante la embriogénesis del paladar junto con otros factores el desarrollo de fisuras faciales (Fig. 1).
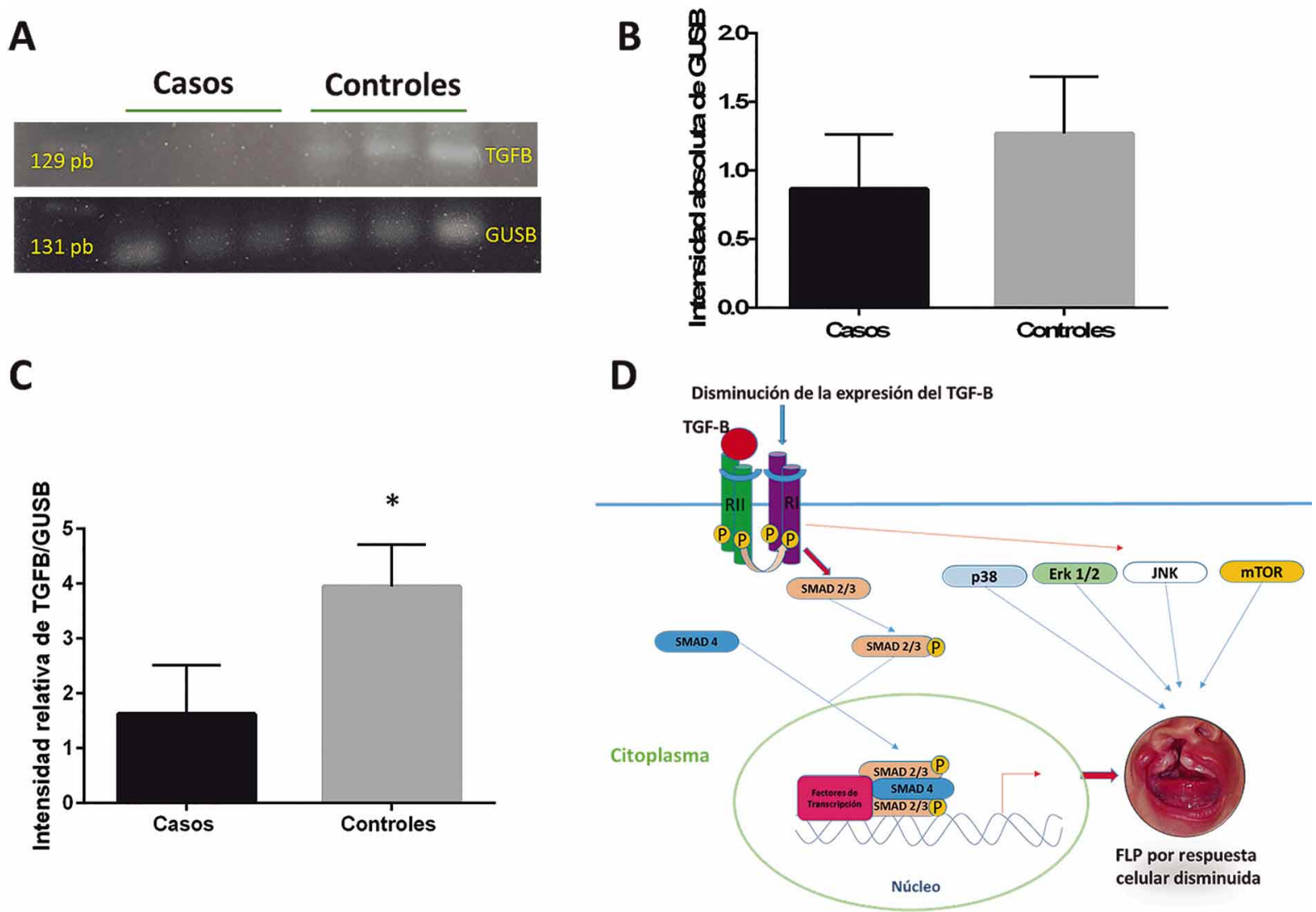

Fig. 1. Comparación de los niveles de expresión génica del TGF-ß. A. Geles de agarosa al 1 \% de la amplificación por PCR del gen TGF-ß y GUSB de niños con FLPNS (casos) y sin FLPNS (controles). B. Niveles de expresión génica de GUSB por grupos, Prueba T-Student $(p=0,1157)$. C. Niveles de expresión génica relativa de TGF-ß/GUSB por grupos, Prueba TStudent $(p=0,0205),{ }^{*}$ estadísticamente significativo. D. Diagrama de la vía de señalización del TGF-ß implicada en el desarrollo de FLAPNS, la disminución en la expresión génica del TGF-ß posiblemente disminuye la respuesta celular de esta vía lo que podría generar durante la embriogénesis del paladar el desarrollo de fisuras palatinas (FLP).

\section{DISCUSIÓN}

Este estudio tiene una serie de limitaciones entre las cuales está la temporalidad en la secuencia causa efecto de las variables estudiadas; sin embargo la realización de procedimientos estandarizados y 
la utilización de instrumentos validados permiten arrojar resultados confiables acerca de la expresión génica del TGF-ß y su posible asociación con el desarrollo de las FLPNS.

Con respecto a las características sociodemográficas y la presencia de FLP los resultados sugieren que no existen asociaciones entre estas, sin embargo Brydon et al. (2014), Conway et al. (2015), Singh et al. (2012a, 2012b ) y Ochoa Lozano et al. (2003) afirman que estas malformaciones son más frecuentes en hombres, asimismo Nazer et al. (2001) sostiene que las fisuras de paladar primario, ya sean con paladar secundario o aisladas son más frecuentes en el sexo masculino; por su parte Sacsaquispe \& Ortiz (2004) concluyeron que el labio y paladar fisurado y la fisura del paladar aislado son más frecuentes en varones; mientras que el labio fisurado aislado es más frecuente en el sexo femenino, del mismo modo Martelli et al. (2012) reportó que las fisuras de paladar aislado son más frecuentes en las mujeres, mientras que la fisura completa de labio, paladar y la submucosa predominaron en el sexo masculino. Cabe aclarar que aún no se conoce por qué se presentan estas variaciones en cuanto al sexo, debido a la complejidad de la etiología de estas alteraciones, la cual incluye la interacción de diferentes factores, sin embargo se podría pensar que posiblemente algunas de estas alteraciones están asociadas con una predicción o susceptibilidad por regiones específicas de los cromosomas, las cuales al estar en contacto con algunos factores ambientales y pueden contribuir con el desarrollo de FLP.

Con relación al tipo de FLP se encontró que la mayor frecuencia fue la bilateral completa, es decir aquella que afecta los dos lados faciales comprometiendo tanto el paladar primario como el secundario, en este sentido Yassaei et al. (2010), Suleiman et al. (2005), Doray et al. (2012) y Genisca et al. (2009) coinciden con que las fisuras completa son las más frecuentes, sin embargo este último autor aclara que en este tipo de fisuras la frecuencia de la unilateral es el doble que la bilateral; por su parte Martelli-Júnior et al. (2008) encontraron un mayor predominio de fisura labio palatina completa, ya sea unilateral o bilateral, seguido de fisura submucosa y por ultimo de fisura palatina aislada. Contrario a esto Singh et al. (2012a, 2012b) y Manyama et al. (2011) sostienen que la de mayor prevalencia es la fisura que afecta únicamente el labio. Estas diferencias se deben posiblemente a las características propias de cada una de las poblaciones estudiadas entre los cuales están el área geo- gráfica y los posibles factores ambientales que se encuentren en ella.

Al evaluar los factores posiblemente asociados no se encontraron diferencias significativas con el consumo de alcohol, cigarrillo, medicamentos, enfermedades durante el primer trimestre de embarazo, edad de la madre y padre durante la concepción del niño, lo que concuerda con lo reportado por Beaty et al. (2001) quienes tampoco encontraron asociación con el cigarrillo, uso de vitaminas, infecciones del tracto urinario y consumo de medicamentos, contrario a esto Wyszynski \& Beaty (1996) reportaron que entre los factores de riesgo para el desarrollo de FLP están el cigarrillo, medicamentos antiepilépticos y uso de vitaminas durante el periodo de preconcepción, consumo de alcohol y exposición a agentes químicos utilizados en la agricultura, asimismo Sabbagh et al. (2015) afirman que existe un incremento significativo de estas alteraciones craneofaciales con el uso de antibióticos, medicamentos antieméticos, enfermedades severas, gripe común, cigarrillo e inclusive tabaquismo pasivo, además sugiere que como factor protector están la suplementación de calcio y el consumo de agua potable con alto contenido de minerales por parte de la madre. Por su parte Hao et al. (2015) sostiene que la historia de fiebre de la madre y de resfriado común, el tabaquismo paterno y el consumo de alcohol, la exposición a la madre a disolventes orgánicos, metales pesados o pesticidas y el uso de multivitamínicos durante el periodo preconcepcional se asociaron con FLPNS. Del mismo modo Martelli et al. (2015) solo encontró asociación entre estas y el uso de cigarrillo por parte de la madre; teniendo en cuenta estos resultado aún no está totalmente claro cuáles son los factores ambientales que están relacionados con el desarrollo de FLAP, una de las posibles explicaciones en las variaciones de los reportes encontrados seria las diferencias geográficas y ambientales en la población utilizada en cada estudio.

Con relación a la expresión génica del TGF-ß en niños con FLPNS se encontró una asociación estadísticamente significativas entre estas y el nivel de expresión, en donde los niños casos presentaban una disminución en la expresión de este transcripto, lo que sugiere que alteraciones en la vía de señalización del TGF-ß posiblemente están relacionadas con el desarrollo de defectos congénitos del desarrollo del paladar y labio; teniendo en cuenta el tipo de muestra utilizada en este estudio y los análisis realizados, estos resultados pueden ser considerados con innovadores debido a que no se encontraron reportes 
en la literatura que evaluaran la expresión génica del transcripto TGF-ß y su asociación con las FLPNS en muestra de saliva, sin embargo se encontraron algunos reportes que lo hicieron en otro tipo de muestra, entre estos el realizado por Baroni et al. (2010) quienes compararon la expresión génica del TGF-ß en fibroblastos aislados del paladar de individuos con FLP sugiriendo que existen una mayor expresión de este transcripto en estos sujetos, asimismo estos mismo autores (Baroni et al., 2006) evaluaron la expresión génica de varios transcriptos posiblemente implicados en FLP en cultivos celulares de fibroblastos obtenidos del paladar secundario de niños con FLPNS y expuestos a diferentes sustancias, sugiriendo que los fibroblastos de FLAP en comparación con fibroblastos normales exhiben un fenotipo anormal in vitro y responden de manera diferente al tratamiento con ácido retinoico, además afirma que la vía de señalización de TGF-ß podría estar implicada con el fenotipo de paladar fisurado. Cabe aclarar que los diseños de los ensayos empleados en estos estudios hacen que sus resultados no sean del todo comparables, sin embargo se podría afirmar que aunque no esté claro el mecanismo, de alguna manera el TGF- $\beta$ está relacionado con la etiología de estas fisuras. Entre los posibles mecanismos por medio del cual el TGF-ß está involucrado en el desarrollo de FLPNS, se ha reportado alteraciones en su vía de señalización durante la palatogénesis, siendo este un proceso complejo que involucra múltiples vías de señalización y varias interacciones entre las células mesenquimales y epiteliales (Meng et al.), entre los pasos críticos en la palatogénesis está el crecimiento, la alineación y fusión de las crestas palatinas, que se completa a finales del primer trimestre en los seres humanos (Bush \& Jiang, 2012); estas estructuras palatinas se componen de un grupo diverso de células derivadas de la cresta neural craneal, mesodermo y células epiteliales derivadas del ectodermo (Iwata et al., 2011). En este sentido la vía de señalización del TGF$ß$ tiene una profunda influencia en los perfiles de expresión génica de células de la cresta neural craneal y su destino de desarrollo (Iwata et al.). Asimismo teniendo en cuenta que las proteínas ligandos de esta vía, específicamente TGF-ß1, TGF-ß2 y TGF-ß3 al ser secretadas son las que inician la cascada de señalización a través de los complejos de receptores de la superficie celular TGFßR1 y TGFßR2, se ha planteado que el TGF-ß1, TGF-ß2 participan principalmente en la regulación de la proliferación del mesénquima palatal mientras TGF-ß3 ha sido propuesto para desempeñar un papel crucial en la regulación del destino de la línea media del paladar a tra- vés de las células epiteliales durante la fusión del paladar (Meng et al.). Asimismo se ha sugerido que individuos con mutaciones deletéreas en los genes TGFßR1 o TGFßR2 desarrollan Síndrome de LoeysDietz, una enfermedad autosómica recesiva normalmente caracterizado por afecciones a nivel vascular, esquelético, y craneofacial, incluyendo fisura palatina (Loeys et al., 2005; Pezzini et al., 2012).

Por otro lado se ha sugerido que la actividad de señalización del TGF-ß está íntimamente relacionada con la matriz extracelular, en donde concentraciones de algunos componentes de esta pueden inactivar al ligando TGF- $\beta$, formando un complejo de unión no covalente en algunas regiones, lo que podría estar influenciado por la biodisponibilidad o función de algunos activadores del TGF-ß (Doyle et al., 2012). Además, se ha sugerido que la matriz extracelular podría influir en la actividad de los efectores de la superficie celular de la señalización del TGF-ß (Doyle et al., 2012). A través de la retroalimentación de modulación, la señalización del TGF-ß puede tener un profundo impacto en la matriz extracelular mediante la estimulación de la síntesis de componentes de los proteoglicanos, colágenos y glucoproteínas, inhibiendo proteasas implicadas en su degradación, y la alteración de las proporciones de la relación de receptores de superficie celular que podrían facilitar la adhesión a la matriz extracelular (Pelikan et al., 2013).

En conclusión los factores de riesgo evaluados mostraron poca evidencia de asociación con la presencia de FLPNS. Los niños con FLPNS tienen menor expresión génica del TGF- $ß$, sugiriendo que alteraciones moleculares en la vía de señalización del TGF-ß posiblemente están involucradas en el desarrollo de las fisuras labio palatinas, ya que se pueden afectar procesos de diferenciación, crecimiento y proliferación celular, en donde participan varios genes incluyendo el TGF-ß.

\section{AGRADECIMIENTOS}

Los autores agradecen a las directivas de la Facultad de Medicina de la Universidad de Cartagena, a COLCIENCIAS por su apoyo con la Financiación a través del programa de Jóvenes Investigadores y a la Dra. Lesbia Rosa Tirado Amador, investigadora de la Facultad de Odontología de la Universidad de Cartagena por su apoyo invaluable. 
MADERA, A. M. V.; GONZÁLEZ, M. F.; ROMERO, S. D. E. \& SUÁREZ, C. A. Gene expression of Transforming Growth Factor Beta in children with non-syndromic cleft lip and palate. Int. J. Odontostomat., 10(1):75-84, 2016.

ABSTRACT: The aim of this study was to determine gene expression of transforming growth factor beta (TGF- $ß$ ) and possible risk factors in children with and without non-syndromic oral clefts. Cases (Children with cleft lip and palate; $n=20$ ) and controls (unaffected children; $n=40$ ) study, from saliva samples mRNA extraction was performed using the RNeasy ${ }^{\circledR}$ Protect Saliva Mini Kit-QIAgen and gene expression of TGF- $B$ by reverse transcriptase polymerase chain reaction, a questionnaire was also applied to record sociodemographic characteristics and possible risk factors. Data were analyzed using measures of central tendency and dispersion, frequencies and percentages. Odds ratio, Chi-square and T-Student tests were used to determine association $(p<0.05)$. The average age of participants was $6.8(S D=4.6)$ years and $63.3 \%$ were male. No statistically significant association was found between any of the possible risk factors examined with the development of oral clefts ( $p>0.05$ ), however it was reported that $11.7 \%$ of mothers had ingested some type of drug during pregnancy, $1.7 \%$ reported maternal smoking and $13.3 \%$ reported alcohol consumption. There were significant differences in gene expression levels of TGF- $\beta$ between groups $(p=0.0205)$, being lower in children with oral clefts. Most environmental risk factors evaluated here gave little evidence of association with the presence of oral clefts. Children with oral clefts have lower gene expression of TGF- $\beta$, suggesting that molecular alterations in the signaling pathway of TGF- $\beta$ are possibly involved in the development of cleft lip and palate, as they can affect processes of differentiation, growth and proliferation cell, where several genes are involved including TGF-ß.

KEY WORDS: cleft lip and palate, Transforming Growth Factor beta, signal transduction.

\section{REFERENCIAS BIBLIOGRÁFICAS}

Ajike, S. O.; Adebola, R. A.; Efunkoya, A.; Adeoye, J.; Akitoye, O. \& Veror, N. Epidemiology of adult cleft patients in North-western Nigeria: our experience. Ann. Afr. Med., 12(1):11-5, 2013.

Aminpour, S. \& Tollefson, T. T. Recent advances in presurgical molding in cleft lip and palate. Curr. Opin. Otolaryngol. Head Neck Surg., 16(4):339-46, 2008.

Andrieux, G.; Fattet, L.; Le Borgne, M.; Rimokh, R. \& Théret, $N$. Dynamic regulation of Tgf- $\beta$ signaling by Tif1y: a computational approach. PLoS One, 7(3):e33761, 2012.

Baroni, T.; Bellucci, C.; Bellucci, C.; Lilli, C.; Pezzetti, F.; Carinci, F.; Becchetti, E.; Carinci, P.; Stabellini, G.; Calvitti, M.; Lumare, E. \& Bodo, M. Retinoic acid, GABAergic, and TGF-beta signaling systems are involved in human cleft palate fibroblast phenotype. Mol. Med., 12(9-10):237-45, 2006.

Baroni, T.; Bellucci, C.; Lilli, C.; Pezzetti, F.; Carinci, F.; Lumare, E.; Palmieri, A.; Stabellini, G. \& Bodo, M. Human cleft lip and palate fibroblasts and normal nicotine-treated fibroblasts show altered in vitro expressions of genes related to molecular signaling pathways and extracellular matrix metabolism. J. Cell. Physiol., 222(3):748-56, 2010.

Beaty, T. H.; Wang, H.; Hetmanski, J. B.; Fan, Y. T.; Zeiger, J. S.; Liang, K. Y.; Chiu, Y. F.; Vanderkolk, C. A.; Seifert, K. C.; Wulfsberg, E. A.; Raymond, G.; Panny, S. R. \& Mclntosh, I. A case-control study of nonsyndromic oral clefts in Maryland. Ann. Epidemiol., 11(6):434-42, 2001.
Berbert-Campos, C. Legal considerations in the management of cleft lip and palate. Cleft Palate Craniofac J., 44(2):223-5, 2007.

Borno, H. T.; Hussein, E. A.; Dudin, A. \& van Aalst, J. A. Incidence of cleft lip and palate in the palestinian territories: a retrospective study from the Makassed Hospital neonatal unit. Cleft Palate Craniofac. J., 51(4):472-5, 2014.

Brydon, C. A.; Conway, J.; Kling, R.; Mehta, L.; Jabs, E. W. \& Taub, P. J. Cleft lip and/or palate: one organization's experience with more than a quarter million surgeries during the past decade. J. Craniofac. Surg., 25(5):16019, 2014.

Bush, J. O. \& Jiang, R. Palatogenesis: morphogenetic and molecular mechanisms of secondary palate development. Development, 139(2):231-43, 2012.

Cobourne, M. T. The complex genetics of cleft lip and palate. Eur. J. Orthod., 26(1):7-16, 2004.

Conway, J. C.; Taub, P. J.; Kling, R.; Oberoi, K.; Doucette, J. \& Jabs, E. W. Ten-year experience of more than 35,000 orofacial clefts in Africa. B. M. C. Pediatr., 15:8, 2015.

Cooper-Brown, L.; Copeland, S.; Dailey, S.; Downey, D.; Petersen, M. C.; Stimson, C. \& Van Dyke, D. C. Feeding and swallowing dysfunction in genetic syndromes. Dev. Disabil. Res. Rev., 14(2):147-57, 2008. 
Costello, B. J. \& Ruiz, R. L. Unilateral cleft lip and nasal repair: the rotation-advancement flap technique. Atlas Oral Maxillofac. Surg. Clin. North Am., 17(2):103-16, 2009.

Chai, Y. \& Maxson, R. E. Jr. Recent advances in craniofacial morphogenesis. Dev. Dyn.,235(9):2353-75, 2006.

Chen, L. J.; Messner, A. H. \& Curtin, G. Newborn hearing screening in infants with cleft palates. Otol. Neurotol., 29(6):812-5, 2008.

Chen, Y.; Dabovic, B.; Annes, J. P. \& Rifkin, D. B. Latent TGF-beta binding protein-3 (LTBP-3) requires binding to TGF-beta for secretion. F. E. B. S. Lett., 517(1-3):27780, 2002.

Doray, B.; Badila-Timbolschi, D.; Schaefer, E.; Fattori, D.; Monga, B.; Dott, B.; Favre, R.; Kohler, M.; Nisand, I.; Viville, B.; Kauffmann, I.; Bruant-Rodier, C.; Grollemund, B.; Rinkenbach, R.; Astruc, D.; Gasser, B.; Lindner, V.; Marcellin, L.; Flori, E.; Girard-Lemaire, F. \& Dollfus, H. Epidemiology of orofacial clefts (1995-2006) in France (Congenital Malformations of Alsace Registry). Arch. Pediatr., 19(10):1021-9, 2012.

Doyle, J. J.; Gerber, E. E. \& Dietz, H. C. Matrix-dependent perturbation of TGFß signaling and disease. F. E. B. S. Lett., 586(14):2003-15, 2012.

Genisca, A. E.; Frías, J. L.; Broussard, C. S.; Honein, M. A.; Lammer, E. J.; Moore, C. A.; Shaw, G. M.; Murray, J. C.; Yang, W.; Rasmussen, S. A. \& National Birth Defects Prevention Study. Orofacial clefts in the National Birth Defects Prevention Study, 1997-2004. Am. J. Med. Genet. A, 149A(6):1149-58, 2009.

Hao, Y.; Tian, S.; Jiao, X.; Mi, N.; Zhang, B.; Song, T.; An, L.; Zheng, X. \& Zhuang, D. Association of Parental Environmental Exposures and Supplementation Intake with Risk of Nonsyndromic Orofacial Clefts: A Case-Control Study in Heilongjiang Province, China. Nutrients, 7(9):7172-84, 2015.

Iwata, J.; Parada, C. \& Chai, Y. The mechanism of TGF-ß signaling during palate development. Oral Dis., 17(8):733-44, 2011.

Jugessur, A.; Farlie, P. G. \& Kilpatrick, N. The genetics of isolated orofacial clefts: from genotypes to subphenotypes. Oral Dis., 15(7):437-53, 2009.

Kang, J. S.; Liu, C. \& Derynck, R. New regulatory mechanisms of TGF-beta receptor function. Trends Cell Biol., 19(8):385-94, 2009.

Kernahan, D. A. \& Stark, R. B. A new classification for cleft lip and cleft palate. Plast. Reconstr. Surg. Transplant. Bull., 22(5):435-41, 1958.
Kruithof-de Julio, M.; Alvarez, M. J.; Galli, A.; Chu, J.; Price, S. M.; Califano, A. \& Shen, M. M. Regulation of extraembryonic endoderm stem cell differentiation by Nodal and Cripto signaling. Development, 138(18):3885-95, 2011.

Lidral, A. C.; Moreno, L. M. \& Bullard, S. A. Genetic Factors and Orofacial Clefting. Semin. Orthod., 14(2):103-14, 2008.

Little, J.; Cardy, A. \& Munger, R. G. Tobacco smoking and oral clefts: a meta-analysis. Bull. World Health Organ., 82(3):213-8, 2004.

Loeys, B. L.; Chen, J.; Neptune, E. R.; Judge, D. P.; Podowski, M.; Holm, T.; Meyers, J.; Leitch, C. C.; Katsanis, N.; Sharifi, N.; Xu, F. L.; Myers, L. A.; Spevak, P. J.; Cameron, D. E.; De Backer, J.; Hellemans, J.; Chen, Y.; Davis, E. C.; Webb, C. L.; Kress, W.; Coucke, P.; Rifkin, D. B.; De Paepe, A. M. \& Dietz, H. C. A syndrome of altered cardiovascular, craniofacial, neurocognitive and skeletal development caused by mutations in TGFBR1 or TGFBR2. Nat. Genet., 37(3):275-81, 2005.

Manyama, M.; Rolian, C.; Gilyoma, J.; Magori, C. C.; Mjema, K.; Mazyala, E.; Kimwaga, E. \& Hallgrimsson, B. An assessment of orofacial clefts in Tanzania. B. M. C. Oral Health, 11: 5, 2011.

Martelli-Júnior, H.; Bonan, P. R.; Santos, R. C.; Barbosa, D. R.; Swerts, M. S. \& Coletta, R. D. An epidemiologic study of lip and palate clefts from a Brazilian reference hospital. Quintessence Int., 39(9):749-52, 2008.

Martelli, D. R.; Coletta, R. D.; Oliveira, E. A.; Swerts, M. S.; Rodrigues, L. A.; Oliveira, M. C. \& Martelli Júnior, H. Association between maternal smoking, gender, and cleft lip and palate. Braz. J, Otorhinolaryngol., 81(5):514-9, 2015.

Martelli, D. R.; Machado, R. A.; Swerts, M. S.; Rodrigues, L. A.; Aquino, S. N. \& Martelli Júnior, H. Non syndromic cleft lip and palate: relationship between sex and clinical extension. Braz. J. Otorhinolaryngol., 78(5):116-20, 2012.

Massague, J. \& Wotton, D. Transcriptional control by the TGFbeta/Smad signaling system. E. M. B. O. J., 19(8):174554, 2000.

Mazzieri, R.; Jurukovski, V.; Obata, H.; Sung, J.; Platt, A.; Annes, E.; Karaman-Jurukovska, N.; Gleizes, P. E. \& Rifkin, D. B. Expression of truncated latent TGF-betabinding protein modulates TGF-beta signaling. J. Cell Sci., 118(Pt. 10):2177-87, 2005.

Meng, L.; Bian, Z.; Torensma, R. \& Von den Hoff, J. W. Biological mechanisms in palatogenesis and cleft palate. J. Dent. Res., 88(1):22-33, 2009. 
Mossey, P. A.; Little, J.; Munger, R. G.; Dixon, M. J. \& Shaw, W. C. Cleft lip and palate. Lancet, 374(9703):1773-85, 2009.

Nazer, H. J.; Hubner G., M. E.; Catalán M., J. \& Cifuentes O., L. Incidencia de labio leporino y paladar hendido en la Maternidad del Hospital Clínico de la Universidad de Chile y en las maternidades chilenas participantes en el Estudio Colaborativo Latino Americano de Malformaciones Congénitas (ECLAMC) período 1991-1999. Rev. Méd. Chil., 129(3):285-93, 2001.

Ocak, Z.; Yazicioglu, H. F.; Aygun, M.; Ilter, M. K. \& Ozlu, T. Prenatal detection of Pai syndrome without cleft lip and palate: a case report. Genet. Couns., 24(1):1-5, 2013.

Ochoa Lozano, B. R.; Ortíz de Anda, J. D.; Padilla de la Paz, K. E.; Chacón Martínez, H. \& Blanco Dávila, F. Casuística de 10 años de labio y paladar hendido en el Hospital Universitario de la UANL. Med. Univ., 5(18):19-24, 2003.

Pelikan, R. C.; Iwata, J.; Suzuki, A.; Chai, Y. \& Hacia, J. G. Identification of candidate downstream targets of TGFß signaling during palate development by genome-wide transcript profiling. J. Cell. Biochem., 114(4):796-807, 2013.

Pezzini, A.; Del Zotto, E.; Giossi, A.; Volonghi, I.; Costa, P. \& Padovani, A. Transforming growth factor $B$ signaling perturbation in the Loeys-Dietz syndrome. Curr. Med. Chem., 19(3):454-60, 2012.

Prahl-Andersen, B. \& Ju, Q. Quality improvement of cleft lip and palate treatment. Angle Orthod., 76(2):265-8, 2006.

Prescott, N. J.; Winter, R. M. \& Malcolm, S. Nonsyndromic cleft lip and palate: complex genetics and environmental effects. Ann. Hum. Genet., 65(Pt. 6):505-15, 2001.

Qian, X.; Jin, L. \& Lloyd, R. V. TGF-B and Estrogen Regulate P27(Kip1) and Cyclin D(1) in Normal and Neoplastic Rat Pituitary Cells. Endocr. Pathol., 8(3):241-50, 1997.

Sabbagh, H. J.; Alamoudi, N. M.; Abdulhameed, F. D.; Innes, N. P.; Al-Aama, J. Y.; Hummaida, T.; Almalik, M.; El Derwi, D. A. \& Mossey, P. A. Environmental risk factors in the etiology of nonsyndromic orofacial clefts in the Western Region of Saudi Arabia. Cleft Palate Craniofac. J., doi: 10.1597/14-136, 2015.

Sacsaquisp, C. S. \& Ortiz, L. Prevalencia de labio y/o paladar fisurado y factores de riesgo. Rev. Estomatol. Herediana, 14(1-2):54-8, 2004.

Sakaki-Yumoto, M.; Katsuno, Y. \& Derynck, R. TGF-beta family signaling in stem cells. Biochim. Biophys. Acta, 1830(2):2280-96, 2013.
Scapoli, L.; Martinelli, M.; Arlotti, M.; Palmieri, A.; Masiero, E.; Pezzetti, F. \& Carinci, F. Genes causing clefting syndromes as candidates for non-syndromic cleft lip with or without cleft palate: a family-based association study. Eur. J. Oral Sci., 116(6):507-11, 2008.

Shen, M. M. Nodal signaling: developmental roles and regulation. Development, 134(6):1023-34, 2007.

Singh, V. P.; Sagtani, R. \& Sagtani, A. Prevalence of cleft lip and cleft palate in a tertiary hospital in Eastern Nepal. Mymensingh Med. J., 21(1):151-4, 2012a.

Singh, V. P.; Sharma, J. N.; Roy, D. K. \& Roy, R. K. A study of orofacial clefts seen in a tertiary referral hospital in Nepal. Ceylon Med. J., 57(2):84-5, 2012 b.

Suleiman, A. M.; Hamzah, S. T.; Abusalab, M. A. \& Samaan, K. T. Prevalence of cleft lip and palate in a hospital-based population in the Sudan. Int. J. Paediatr. Dent., 15(3):1859, 2005.

Tsai, V. W.; Macia, L.; Johnen, H.; Kuffner, T.; Manadhar, R.; Jørgensen, S. B.; Lee-Ng, K. K.; Zhang, H. P.; Wu, L.; Marquis, C. P.; Jiang, L.; Husaini, Y.; Lin, S.; Herzog, H.; Brown, D. A.; Sainsbury, A. \& Breit, S. N. TGF-b superfamily cytokine MIC-1/GDF15 is a physiological appetite and body weight regulator. PLoS One, 8(2):e55174, 2013.

Wong, F. K. \& Hagg, U. An update on the aetiology of orofacial clefts. Hong Kong Med. J., 10(5):331-6, 2004.

Wyszynski, D. F. \& Beaty, T. H. Review of the role of potential teratogens in the origin of human nonsyndromic oral clefts. Teratology, 53(5):309-17, 1996.

Yassaei, S.; Mehrgerdy, Z. \& Zareshahi, G. Prevalence of cleft lip and palate in births from 2003 - 2006 in Iran. Community Dent. Health, 27(2):118-21, 2010.

Dirección para Correspondencia:

Amileth Suárez Causado

Universidad de Cartagena

Campus de la Salud, Zaragocilla

Facultad de Medicina, Departamento de Ciencias Básicas Unidad de Bioquímica

Cartagena

COLOMBIA

Email: asuarezc1@unicartagena.edu.com

Recibido: 21-10-2015

Aceptado: 29-02-2016 